\title{
Overview Of Context-aware Recommender System Research
}

\author{
Haohan Liu, Hongli Zhang*, Kanghua Hui, Huaiqing He \\ Department of Computer Science and Technology, Civil Aviation University of China, Tianjin, China \\ Email: hlzhang1006@163.com
}

\begin{abstract}
Keywords: personalized recommendation; collaborative filtering; content-based recommendation; network-based architecture recommendatio; context-aware recommendation.

Abstract. The traditional recommendation system usually ignored the contextual information. However, there are usually various factors influencing user's decision. Therefore, the incorporation of contextual information in the recommendation process has attracted major interest. In this paper, firstly, the classical collaborative filtering, content-based, network-based architecture and hybrid recommender system research since 2008 are described. Secondly, the research in context-aware recommender system are mainly described, then its characteristics and limitations are analyzed. Finally, the problems and challenges of context-aware recommender system are summarized, and some improved methods and possible future research directions are put forward.
\end{abstract}

\section{Introduction}

In the current era of rapid growth in the amount of information, it takes much more time for us to find the information of great value from mass information. This phenomenon is called "Information Overload". It is important for users and e-commerce businesses to acquire the valuable information efficiently and quickly from mass data. The purpose of personalized recommendation system is to enhance the quality of service and to recommend information or products to users which they may be interest in.

The quality of personalized recommendation system performance depends on which recommendation algorithm is used. Zhou Tao analyzed several common recommendation algorithms from 1990s to 2008 thoroughly in literature [1], these algorithms include content-based, collaborative filtering, network based and hybrid recommendation algorithm.

Firstly, this paper tracks the above four recommendation algorithms' developments since 2008, then it describes a new research in context-aware recommendation system in detail, points out its advantages in addressing the limitations of the traditional recommendation systems, and analyzes the difficulties and challenges which it may face up with, finally points out possible solutions and proposes a number of possible future research directions.

\section{The brief introduction of four types of recommender system}

\section{Content-based recommender system}

Content-based recommender system mainly recommends an item to a user based upon a description of the item and a profile of the user's interests.

The advantages of content-based recommender system were summarized in paper [1], but it also has some limitations, mainly manifested in the following two aspects: (a) Limitation of content analysis (b) Specialization [2].

As was mentioned in [1], most of the methods of content-based recommendation system recommend by analyzing the content information of the product. The most common used method is TF-IDF. On the issues of specialization, literature [3] adopts the the semantic Web reasoning technology to deduct the semantic association between the user preference and product. To the question of data sparsity, [4] introduce Bayesian theory to extract the characteristics of the product and the user. During the process of user's interest modeling, [5] analyzed the fuzzy semantics and described the acquisition methods of invisible data. 
Cui Chunsheng pointed out two challenges the system faced in [6]. One is that the system only recommend the product in accordance with the original interest to the user; Another is the extraction of the user and product feature.

\section{Collaborative Filtering Recommender system}

The main idea of the collaboration filtering recommendation is to use the existing user's past behavior or comments to provide the product which conforms with the current user's requirements.

The basic principle of the system is to find the neighbor user who has the similar past purchase behavior with the target user. Then use these neighbors' purchase behavior to recommend to the target user[7].

In the process of recommendation, the most used methods to form the neighbor users set include Top-N, K nearest method, threshold method, clustering and Bayesian networks method. Similarity measure methods include the cosine similarity, modified cosine similarity and correlation similarity and entropy. Forecast generated methods include simple average, weighted average, center weighted average and standard weighted average[7].

In addition to the typical problem of data sparsity and extensible, there are some new problems as follows: (a) interest migration; (b)fuzzy users; (c) accuracy.

In order to solve the problem of cold start and sparsity, literature [8] adopted the clustering algorithm to classify all products. Literature [9] adopted the combined clustering algorithm to reduce the dimension of the raw data.

To the question of inaccurate in finding the user's nearest neighbors. Literature [10] put forward an algorithm which combines the conditional probability and non-fixed $\mathrm{j}$ nearest algorithm. Literature [11] proposed a method which calculates the proportion between the common ratings and all the ratings.

In view of the traditional collaborative filtering can only consider limited data when calculating the similarity between users. Literature [12] put forward a user interest estimation model based on kernel density estimation.From the perspective of dynamic recommendation, literature [13] proposed a time weighted uncertain neighbor collaborative filtering algorithm.

\section{Network-based recommender system}

Network-based recommender algorithm is a new kind of algorithm based on resource allocation, the key to the algorithm is to establish user-product binary association network.

Literature [1] elaborated the recommendation algorithm based on binary resource allocation in detail, it analyzed the impact of the product's degree on recommendation accuracy. It also proposed an algorithm through wiping out the duplication attribute to acquire a higher precision, and improve the algorithm accuracy by introducing the coupling threshold as well as reduce the algorithm complexity.

Network based recommendation algorithm mainly includes three categories: resource allocation, heat conduction and diffusion.

To solve the problem of new user and item of network based recommendation system, literature [14] considered the characteristics similarity of the item in the process of network structure construction. Due to the original non-value binary network, literature [15] pointed out that user-item binary network edge weight should be considered. According to user's interests will shift over time, literature [19] proposed an improved recommendation algorithm based on time weight.

\section{Hybrid recommender system}

From the above three sections, we can find that the existing recommendation systems have their own limitations in the recommendation process. Many scholars have put forward hybrid recommendation which fuses various recommendation algorithms together.

There are a lot of hybrid recommendation methods in theory, in addition to the hybrid methods described in literature [1]. According to different application scenarios, there are mainly two kinds of hybrid methods as follows:

Hybrid of recommended results: this method adopts a certain algorithm to fuse each recommended result, the final result depends on the mix of each recommended result of recommendation system. 
Hybrid of recommendation algorithm: integrate other recommendation algorithms into one algorithm to realize recommendation.

\section{Context-aware recommender system}

In order to solve some problems of traditional recommender system, context-aware recommender system has attracted the attention of the academic. Contextual information is an important factor influencing the accuracy of the recommendation. The purpose of the context-aware recommender system is to consider the position, time and some additional information in the process of recommendation.

The means to use the contextual information can mainly be classified into two kinds in recommender system, which are as follows: (a)recommended by context-driven query and retrieval; (b)recommended by context preference extract and evaluation

The recommendation process mainly includes four stages: contextual information acquisition, user preference extraction, context-aware recommendation generated, recommendation evaluation and improvement [20].

\section{Contextual information acquisition}

The accesses of acquiring information are divided into three categories: explicit acquisition, implicit acquisition and reasoning acquisition.

Contextual information may also be hidden in some of the underlying data table, these implicit information can still be deduced by the modeling and machine learning technology, thus improving the accuracy of recommendation.

\section{Contextual user preference extraction}

Preference is the description that how much the user is fond of the item, the process of the preference extraction is to find what the user really likes. Contextual user preference extraction technology mainly has two research ideas: quantitative analysis and qualitative analysis[21].

Quantitative research is to use digital score quantitative signify contextual user preferences. It can be divided into two categories: heuristic based and model based..

Qualitative research focus on the perspective of logical reasoning and partial order model to extract partial order relation of any two specific items and its properties from the user. It mainly uses strict partial order preference extraction technology to extract contextual user preference.

\section{Context-aware recommendation generated}

Context-aware recommendation is recommended on the basis of some known user preferences, combing forecasting potential user preferences. It can be divided into three paradigms: contextual prefiltering, contextual postfiltering and contextual modeling.

\section{Evaluation of context-aware recommender system}

The evaluation index mainly includes recommendation accuracy, diversity, novelty and coverage. The most used index is accuracy. In addition, user satisfaction is also used as an evaluation index of context-aware recommendation system.

\section{Problems and challenges}

Although the research on context-aware recommendation has got great process in recent years, it still faces many problems and challenges, which mainly include following aspects: (a)formal definition; (b) test and calculation of the valid context; (c)technology of contextual user preference extraction; (d)technology of context-aware recommendation generated; (e)utility evaluation; (f)privacy and security issues.

\section{Conclusion and outlook}

This paper summarized the research work of the four recommendation system since 2008 based on the literature [1], and then described the context-aware recommendation system in detail.

In recent years, scholars carry out the research on the context-aware recommender system from different perspectives. Research hotspots and future research directions of the context-aware 
recommender system can be concluded as follows: (a)large-scale context-aware recommender system; (b)Recommender system based on social network; (c)interact with context-aware recommender systems; (d)mobile context-aware recommender system; (e)cross-over study between context-aware recommender system and other subjects.

\section{References}

[1]LIU Jian-guo, ZHOU Tao, WANG Bing-hong. Research progress in personalized recommendation system[J]. Progress In Natural Science, 2009, 19(1): 1-15.

[2]XIA Pei-yong. Research on Collaborative Filtering Algorithm of Personalized Recommendation Technology [D].Ocean University of China, 2011.

[3]Blanco-Fernandez Y, Pazos-Arias J, Gil-Solla A, et al. Providing entertainment by content-based filtering and semantic reasoning in intelligent recommendation systems[J]. Consumer Electronics, IEEE Transactions on, 2008, 54(2): 727-735.

[4]MENG Xian-fu, Chen Li. Collaborative filtering recommender algorithm based on Bayesian theory[J]. Journal of Computer Application, 2009, 29(10): 2733-2735.

[5] LI Xiao-jun, YANG Xiao-hua, YU Ying. Research on individualized recommendation based on implicit feedback analyses[J]. Computer Engineering and Design, 2009 (16): 3794-3796.

[6] CUI Chun-sheng, LI Guang, WU Qi-zong. Research on recommender systems of electric commerce based on Vague sets[J]. Computer Engineering and Application, 2011, 47(10): 237-239.

[7] LU Wei.Collaborative filtering algorithm and its application in personalized recommendation system[D]. Beijing University of Posts and Telecommunication, 2007.

[8]DENG Ai-lin, ZUO Zi-ye, ZHU Yang-yong. Collaborative Filtering Recommendation Algorithm Based on Item Clustering[J]. Mini-micro Systems, 2004, 25(9): 1665-1670.

[9]WU Hu, WANG Yong-ji, WANG Zhe. Two-Phase Collaborative Filtering Algorithm Based on Co-Clustering[J]. Journal of software, 2010, 21(5): 1042-1054.

[10]ZHANG Yu-fang, DAI Jin-long, XIONG Zhong-yang. Collaborative Filtering algorithm based on two-step filling for alleviating data sparsity[J]. Application Research of Computer, 2013, 30(9): 2602-2605.

[11]WEN Jun-hao, SHU Shan. Improved Collaborative Filtering Recommendation Algorithm of Similarity Measure[J]. Computer Science, 2014, 41(5): 68-71.

[12]WANG Peng, WANG Jing-jing, YU Neng-hai. A Kernel and User-Based Collaborative Filtering Recommendation Algorithm[J]. Journal of Computer Research and Development, 2013, 50(7): 1444-1451.

[13]ZHENG Zhi-gao LIU Jing WANG Ping SUN Sheng-li, Time weighted Uncertain Nearest Neighbor Collaborative Filtering Algorithm[J].. Computer Science., 2014, 41(8)

[14]L ü Shan-guo, WU Xiao-kui,CAO Yi-qin, Network-based Recommendation Algroithm[J], research and exploration in laboratroy,2012, 7: 081.

[15]Pan X, Deng G, Liu J G. Weighted bipartite network and personalized recommendation[J]. Physics Procedia, 2010, 3(5): 1867-1876.

[19]DU Han. The Reaseach of Recommendation Algorithm based on Network. Beijing University of Posts and Telecommunications, 2013.

[20]WANG Li-cai, MENG Xiang-wu, ZHANG Yu-jie. Context-Aware Recommender system [J]. Journal of Software, 2012, 23(1). 